\title{
CARACTERIZAÇÃO DE ZEÓLITAS NATURAIS CLINOPTILOLITA
}

\author{
Characterization of Natural zeolites Clinoptilolite
}

\author{
Renata Cibely Freire de Lima ${ }^{1}$; Daniele da Silva Oliveira ${ }^{1}$; Sibele Berenice Castellã Pergher ${ }^{1}$
}

${ }^{1}$ LABPEMOL, Instituto de Química, Universidade Federal do Rio Grande do Norte, Câmpus Universitário, Lagoa Nova, Natal, RN. E-mail: sibelepergher@gmail.com

Data do recebimento: 30/03/2020 - Data do aceite: 01/08/2020

RESUMO: Zeólitas são aluminossilicatos com arranjo estrutural composto por um esqueleto formado pela combinação tridimensional de tetraedros de $\mathrm{AlO}_{4}$ e $\mathrm{SiO}_{4}$, unidos entre si através de átomos de oxigênio. Sua estrutura apresenta canais e cavidades, nas quais se encontram moléculas de água e cátions trocáveis. Quatro zeólitas Clinoptilonita foram utilizadas neste trabalho - duas fornecidas pela empresa Celta Brasil e outras duas provenientes do Chile. A zeólita natural Clinoptilolita $(\mathrm{Si} / \mathrm{Al} \geq 4)$ pertence ao grupo da Heulandita e apresenta seletividade por íons de pequeno raio devido ao tamanho de suas cavidades. Foi realizado um estudo estrutural da Clinoptilolita, antes e após tratamento térmico a $450^{\circ} \mathrm{C}$, visto que a Heulandita e a Clinoptilolita apresentam propriedades físicas e químicas similares. Os materiais foram caracterizados por difração de raios X (DRX), fluorescência de raios X (FRX), análise termogravimétrica (TG/DTG). Foi observado que no processo de calcinação os materiais são essencialmente Clinoptilolita, uma vez que esta fase permaneceu estável à alta temperatura por ser mais resistente, diferentemente da Heulandita. Da mesma forma, os materiais apresentaram composição química referente à fase Clinopilonita. Entretanto, se evidenciou, tanto pelo DRX como pelo FRX, a presença de algumas impurezas, especialmente contendo ferro. Os materiais se mostraram adequados para serem empregados no tratamento de efluentes em que se necessite alta capacidade de troca catiônica, bem como materiais economicamente viáveis e disponíveis na natureza.

Palavras-chave: Zeólita natural. Clinoptilolita. Caracterização.

ABSTRACT: Zeolites are aluminosilicates with a structural arrangement composed of a skeleton formed by the three-dimensional combination of $\mathrm{AlO}_{4}$ 
and $\mathrm{SiO}_{4}$ tetrahedrons bonded by oxygen atoms. Its structure has channels and cavities, in which water molecules and exchangeable cations are found. Four zeolie Clinoptilolites were used in this work, two provided by Celta Brazil and the other two from Chile. The natural zeolite Clinoptilolite ( $\mathrm{Si} / \mathrm{Al} \geq 4)$ belongs to the Heulandite group and presents selectivity for small ray ions due to the size of its cavities. A Clinoptilolite structural study was carried out, before and after heat treatment at $450{ }^{\circ} \mathrm{C}$, since Heulandite and Clinoptilolite have similar physical and chemical properties. The materials were characterized by X-ray diffraction (XRD), X-ray fluorescence (XRD), thermogravimetric analysis (TG/DTG). It was observed that in the calcination process all materials are mainly Clinoptilolite since this phase remained stable at high temperature because it is more resistant, different from Heulandite. Likewise, the materials presentend chemical composition referring to the Clinoptitolite phase. However, the presence of some impurities, especially containing iron, was evidenced by both DRX and FRX. The materials proved to be suitable to be used in the treatment of effluents in which high cation exchange capacity is required, as well as economically viable materials available in nature.

Keywords: Natural zeolite. Clinoptilolite. Characterization.

\section{Introdução}

O nome zeólita tem origem grega, zeo (que ebuli) e lithos (pedra), e foi dado a um grupo de minerais naturais que apresentava adsorção reversível de água. Além dessa propriedade, esse grupo de materiais também apresentava capacidade de troca iônica. As zeólitas são aluminossilicatos que apresentam em sua composição átomos tetraédricos de silício e alumínio, contendo cavidades em forma de canais ou caixas que, comumente, estão ocupados por moléculas de água ou cátions compensadores de carga (GIANNETTO, 1990).

Existem zeólitas naturais e sintéticas, ou seja, não encontradas na natureza e produzidas exclusivamente em laboratório (VIEIRA; RODRIGUES; MARTINS, 2014).

A formação das estruturas zeolíticas na natureza é consequência de processos geológicos em rochas basálticas, vulcânicas e sedimentares. Fatores como temperatura, pressão, atividade de espécies iônicas, pressão da água e tipo de solo influenciam na sua formação (KANG et al., 1997; BLANCO VARELA et al., 2006). Estima-se que a produção anual de zeólita natural esteja na casa de 2,5 a 3,0 milhões de toneladas, sendo que as Clinoptilolitas e as Modernitas apresentam maior valor industrial agregado devido ao grande número de depósitos espalhados por diversas regiões do mundo (ÇULFAZ et al., 2004). A China (1,75 a 2,25 milhões de toneladas), Coréia (175,000 toneladas), Japão (140,000 a 160,000 toneladas), Estados Unidos (65,000 toneladas) e Cuba (35,000 a 45,000 toneladas) são os principais produtores mundiais (BERNARDI et al., 2008).

Segundo Farjallat e Suguio (1966), há registro de Heulandita em lente de arenito intercalada a derrames de basalto da Formação Serra Geral. Dessa forma, podemos encontrar depósitos naturais de zeólitas no Brasil, sendo mais comum na bacia do Paraná e do Parnaíba. 
Tabela I - Fórmula química e os parâmetros de células unitárias para Heulandita e para Clinoptilolita

\begin{tabular}{ccl}
\hline Zeolitas & \multicolumn{1}{c}{ Fórmula Química } & \multicolumn{1}{c}{ Parâmetros de células unitárias } \\
\hline \multirow{2}{*}{ Heulandita } & $(\mathrm{Na}, \mathrm{K}) \mathrm{Ca}_{4}\left(\mathrm{Al}_{8} \mathrm{Si}_{28} \mathrm{O}_{72}\right) \cdot 24 \mathrm{H}_{2} \mathrm{O}$ & $\mathrm{a}=17,70 \AA, \mathrm{b}=17,94 \AA, \mathrm{c}=7,42 \AA$ \\
& & $\beta=116^{\circ} 24^{\circ}$ \\
\multirow{2}{*}{ Clinoptilolita } & $(\mathrm{Na}, \mathrm{K}) 6\left(\mathrm{Al}_{6} \mathrm{Si}_{30} \mathrm{O}_{72}\right) \cdot 20 \mathrm{H}_{2} \mathrm{O}$ & $\mathrm{a}=17,62 \AA, \mathrm{b}=17,91 \AA, \mathrm{c}=7,39 \AA$ \\
& $\beta=116^{\circ} 16^{\circ}$ \\
\hline
\end{tabular}

Fonte: Adaptado de Gianetto,1990.

De acordo com International Zeolite Association (IZA, 2020), até o presente, momento foram registrados 67 zeólitas naturais.

Dentro das zeólitas naturais encontram-se a zeólita Clinoptilolita. Um mineral pertencente ao grupo da Heulandita, grupo composto pelos minerais Clinoptilolita e Heulandita, ambas estruturalmente iguais, apenas se diferenciam na quantidade de alumínio na estrutura cristalina, com uma razão $\mathrm{Si} / \mathrm{Al}<$ 4 para Heulandita e $\mathrm{Si} / \mathrm{Al} \geq 4$ para Clinoptilolita, (ARMBRUSTER; BISH; BOAK, 2001; KOCAK; TASCI; KAYA, 2013; COLELLA; WISE, 2014; SERYOTKIN, 2016). Neste grupo também temos outros minerais naturais, como Stilbita e Brewsterita (ARMBRUSTER; GUNTER, 2001). A Clinoptilolita é uma das espécies de zeólita sedimentar encontrada em maior quantidade na natureza (YELIZ, 2010).

As zeólitas Heulandita e Clinoptilolita apresentam difratogramas com reflexões características bem próximas, sendo assim, impossível a identificação que possa distinguir ambas pela técnica de difração de raios $\mathrm{X}$ (DRX). Independente das similaridades e da dificuldade de diferenciá-las pela técnica difração de raios $X$, atualmente considera-se que são zeólitas distintas, que pertencem ao mesmo grupo. Elas apresentam uma simetria monoclínica e sua densidade varia ligeiramente de $2,198 \mathrm{~g} / \mathrm{cm}^{3}$ para Heulandita e 2,16 g/cm ${ }^{3}$ para Clinoptilolita (GIANETTO, 1990).
A Tabela I apresenta a fórmula química e os parâmetros de células unitárias para Heulandita e para Clinoptilolita, mostrando o quão próximos são esses parâmetros, caracterizando assim essa dificuldade em diferenciar as duas zeólitas entre si (GIANETTO, 1990).

A unidade secundária de construção do grupo da Heulandita é o grupo C4-C4=T1 que, dentro desse grupo, obtemos as SUBS (Unidades Secundarias de Construção) de zeólitas que são iguais da nossa amostra estudada. Sendo assim, temos dentro desse grupo as zeólitas Heulandita, Stilbita, Stellerita, Barrerita, Brewsterita (Gianetto, 1990).

A estrutura porosa desta zeólita é constituída por canais na direção [001] com dimensões $5,5 \times 3,1 \AA$ e $4,1 \times 4,1 \AA$ e um canal na direção [100] com dimensões $3,4 \times$ $2,8 \AA$ A (Figura 1), os quais são paralelos e interconectados de forma perpendicular, dando origem a um material poroso (IZA, 2020).

A uniformidade na estrutura e o alto grau de pureza são determinantes para a grande aplicabilidade das zeólitas sintéticas como catalisadores. Por outro lado, as zeólitas naturais, por possuírem menor pureza, são utilizadas, principalmente, no tratamento de efluentes, devido à sua grande capacidade de troca catiônica (BRAGA et al., 2007).

Neste trabalho foram utilizados quatro tipos de Clinoptilolita naturais com o objetivo de caracterizar estes materiais para uma futura aplicação. 
Figura 1 - A) Estrutura HEU vista na direção [001], B) Estrutura HEU vista na direção [100], C) Anel de 10 MR visto no plano [001], D) Anel de 8 MR visto no plano [001] e E) Anel de 8 MR visto no plano [100].

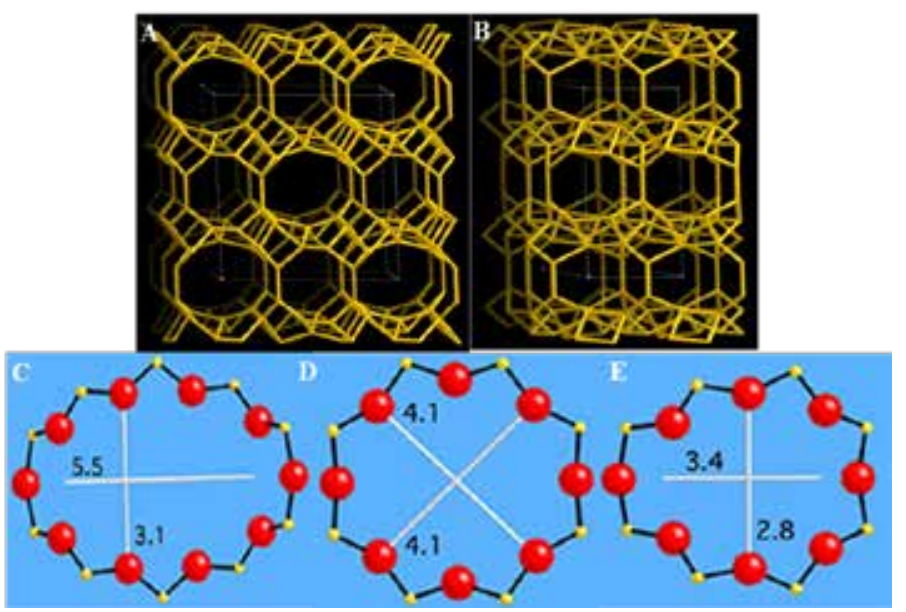

Fonte: Adaptado de www.iza-structure.org.

\section{Materiais e métodos}

Nesse trabalho foram utilizados quatro tipos de Clinoptilolita, codificados com a seguinte nomenclatura: RCB LOT 216, RCB LOT 221, RCN1, RCN2. A RCB foi fornecida pela Celta Brasil e a RCN é proveniente do Chile.

\section{Técnicas de Caracterização}

\section{Calcinação}

Devido as zeólitas Heulandita e Clinoptilolita apresentarem propriedades físicas e químicas similares, para diferenciá-las foi utilizado o tratamento térmico em mufla, a $450{ }^{\circ} \mathrm{C}$, durante 12 horas seguido por análise de difração de raios X (DRX).

\section{Difração de raios $X(D R X)$}

A difração de raios $\mathrm{X}(\mathrm{DRX})$ é uma técnica importante na caracterização estrutural de materiais cristalinos, como é o caso das zeólitas. Neste trabalho, foi empregando o método do pó, em um equipamento modelo Bruker D2 Phaser equipado com um detector Lynxeye aplicando radiações de $\mathrm{CuK} \alpha$ $(\lambda=1,54 \AA)$ com um filtro de $\mathrm{Ni}$, em uma voltagem de $30 \mathrm{kV}$ e corrente de $10 \mathrm{~mA}$. A abertura da fenda divergente foi de $0,6 \mathrm{~mm}$ e da fenda central de $1 \mathrm{~mm}$, passo de $0,02^{\circ}$, sendo que os dados foram coletados em alto ângulo, em uma faixa de graus $2 \theta$ de 5 a $50^{\circ}$, com tempo de aquisição de $0,1 \mathrm{~s}$.

\section{Espectrometria de fluorescência de raios $X(F R X)$}

A análise química foi realizada através da técnica de espectrometria de fluorescência de raios $\mathrm{X}$ (FRX), onde um feixe de elétrons emitidos através de uma fonte catódica permitiu a identificação de espécies atômicas. Esta análise foi realizada em um aparelho Bruker S2 Ranger utilizando radiação Pd ou $\mathrm{Ag}$ anode max. potência de $50 \mathrm{~W}$, max. voltagem $50 \mathrm{kV}$, max. Corrente $2 \mathrm{~mA}$, XFlash ${ }^{\circledR}$ Silicon Drift Detector. 


\section{Análise Termogravimétrica (TG/DTG)}

Através da análise termogravimétrica em zeólita é possível determinar a sua estabilidade térmica, assim como se há a presença de compostos como água, orgânicos, entre outros. Esta análise foi realizada usando uma termobalança modelo TGA 50 da Shimadzo, iniciando-se a $25{ }^{\circ} \mathrm{C}$ e indo até $900{ }^{\circ} \mathrm{C}$, a uma razão de aquecimento $10{ }^{\circ} \mathrm{C} / \mathrm{min}$, sob atmosfera inerte de nitrogênio. Foi utilizado cadinho de platina e $10 \mathrm{mg}$ da zeólita. As perdas de massa da amostra foram calculadas através da derivada primeira da curva termogravimétrica (DTG).

\section{Resultado e discussão}

Para comprovar a estrutura cristalina dessas zeólitas, fez-se uso do banco de dados da International Zeolite Association - IZA. Essas zeólitas apresentaram fases cristalinas da HEU (fase predominante), de acordo com a posição dos seus picos comparados ao padrão da IZA. Através dos difratogramas das zeólitas, pode-se observar na Figura 2 e 3, que não ocorreu alteração significativa nas estruturas das zeólitas após a calcinação (apenas uma

Figura 2 - Difratogramas das amostras antes da calcinação

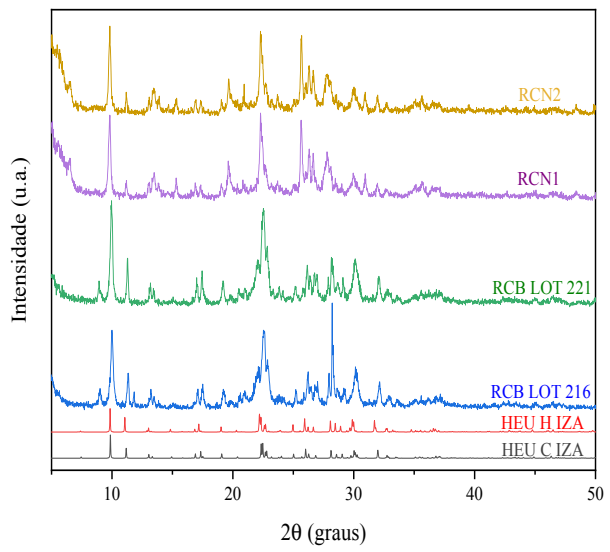

leve diminuição da intensidade de alguns picos), sendo um indicativo que essas zeólitas são Clinoptilolita. Caso houvesse perda de cristalinidade significativa seria referente a presença da Heulandita (GIANNETO, 1990).

Observa-se nas amostras RCN1 e RCN2 uma reflexão a, aproximadamente, $7^{\circ}$; nas amostras RCB LOT 221 e RCB LOT16 aparecem, uma reflexão a, aproximadamente, $9^{\circ}$, entre outras de pequena intensidade. Isto ocorre, provavelmente, devido à presença de impurezas nas amostras, uma vez que são materiais naturais. Mais estudos seriam necessários para descobrir que impurezas seriam.

Como as amostras analisadas se tratam de aluminossilicatos, o sílicio e o alumínio foram uns dos principais elementos da composição dessas zeólitas, como pode ser observado através dos resultados das Tabela II a V.

A amostra estudada por Mansouri et al. (2013), mostra que $\mathrm{SiO}_{2}, \mathrm{Al}_{2} \mathrm{O}_{3}$ e $\mathrm{CaO}$ são os componentes principais da Clinoptilolita. Resultados semelhantes foram observados nesse trabalho. Entretanto, se observaram também a presença de $\mathrm{K}, \mathrm{Fe}, \mathrm{Mg}$, $\mathrm{Na}$ que podem estar compensando carga na estrutura zeolítica ou podem estar presentes nas impurezas.

Figura 3 - Difratogramas das amostras após a calcinação

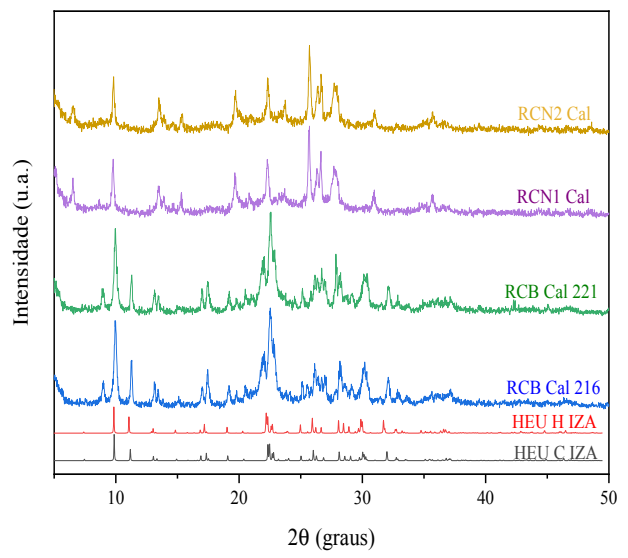


Na Tabela II e III pode-se observar que, após a calcinação, as amostras mantiveram a composição química semelhante a das amostras não calcinada. Esse resultado corrobora com os resultados de DRX, sendo um indicativo que a calcinação não alterou a estrutura e nem a composição dessas amostras.

Observa-se que as amostras provenientes do Chile (RCN1 e RCN2) possuem maior quantidade de ferro que as amostras fornecidas pela Celta Brasil. Isto pode ocorrer por serem materiais naturais provenientes de diferentes localidades e, com isso, outras fases (outros minerais) estariam presentes.

A Clinoptilolita exibe uma ampla quantidade de alumínio em sua estrutura, o que gera uma grande quantidade de carga negativa que precisa de vários íons compensadores de carga. Tais íons compensadores podem ocasionar problema difusional, já que as cavidades do material estão preenchidas e com espaço disponível reduzido. Os principais cátions presentes na Clinoptilolita são: $\mathrm{Ca}^{2+}$, $\mathrm{Mg}^{2+}, \mathrm{Na}^{+}, \mathrm{K}^{+}$e sua distribuição podem ser vistas na Figura 4. Os canais e poros existentes também podem conter água (IZA, 2020).

Tabela II - Composição química (FRX) das amostras antes da calcinação

\begin{tabular}{ccccc}
\hline Óxido & RCB LOT 216 & RCB LOT 221 & RCN1 & RCN2 \\
\hline $\mathrm{SiO}_{2}$ & $68,09 \%$ & $72,29 \%$ & $68,34 \%$ & $66,70 \%$ \\
$\mathrm{Al}_{2} \mathrm{O}_{3}$ & $11,79 \%$ & $12,80 \%$ & $14,58 \%$ & $15,16 \%$ \\
$\mathrm{~K}_{2} \mathrm{O}$ & $5,08 \%$ & $4,47 \%$ & $1,22 \%$ & $1,19 \%$ \\
$\mathrm{CaO}$ & $4,49 \%$ & $4,09 \%$ & $4,93 \%$ & $4,98 \%$ \\
$\mathrm{SO}_{3}$ & $3,88 \%$ & $0,12 \%$ & $0,11 \%$ & $0,14 \%$ \\
$\mathrm{Fe}_{2} \mathrm{O}_{3}$ & $2,19 \%$ & $2,30 \%$ & $6,12 \%$ & $6,30 \%$ \\
$\mathrm{MgO}$ & $1,70 \%$ & $2,10 \%$ & $2,00 \%$ & $2,40 \%$ \\
$\mathrm{Na}_{2} \mathrm{O}$ & $1,40 \%$ & $1,30 \%$ & $1,70 \%$ & $2,10 \%$ \\
$\mathrm{Outros}$ & $1,38 \%$ & $0,53 \%$ & $1,00 \%$ & $1,03 \%$ \\
\hline
\end{tabular}

Tabela III - Composição química (FRX) das amostras após a calcinação

\begin{tabular}{ccccc}
\hline Óxido & RCB LOT 216 & RCB LOT 221 & RCN1 & RCN2 \\
\hline $\mathbf{S i O}_{2}$ & $70,02 \%$ & $72,81 \%$ & $68,44 \%$ & $68,44 \%$ \\
$\mathbf{A l}_{2} \mathbf{O}_{3}$ & $12,18 \%$ & $12,54 \%$ & $14,65 \%$ & $14,65 \%$ \\
$\mathbf{K}_{2} \mathbf{O}$ & $4,96 \%$ & $4,39 \%$ & $1,27 \%$ & $1,27 \%$ \\
$\mathbf{C a O}$ & $3,81 \%$ & $4,04 \%$ & $4,89 \%$ & $4,89 \%$ \\
$\mathbf{S O}_{3}$ & $2,83 \%$ & $0,13 \%$ & $0,12 \%$ & $0,12 \%$ \\
$\mathbf{F e}_{2} \mathbf{O}_{3}$ & $2,04 \%$ & $2,25 \%$ & $5,95 \%$ & $5,95 \%$ \\
$\mathbf{M g O}$ & $1,80 \%$ & $2,0 \%$ & $2,0 \%$ & $2,0 \%$ \\
$\mathbf{N a}_{2} \mathbf{O}$ & $1,20 \%$ & $1,2 \%$ & $1,7 \%$ & $1,7 \%$ \\
$\mathbf{O u t r o s}$ & $1,24 \%$ & $0,57 \%$ & $0,98 \%$ & $0,98 \%$ \\
\hline
\end{tabular}


Figura 4 - Representação de cátions e água nos canais e poros da Clinoptilolita

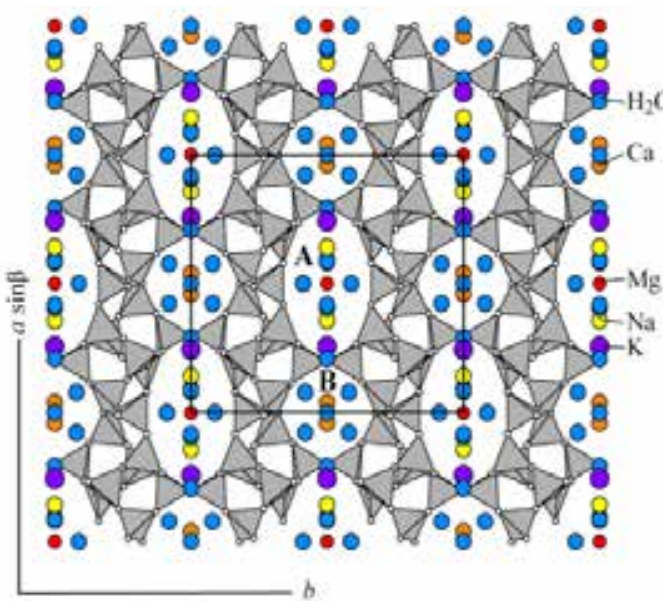

Fonte: www.iza-online.org.

Segundo Ribeiro e Rodrigues (2010), a Clinoptilolita apresenta uma razão $\mathrm{Si} / \mathrm{Al}$ entre 4,25 e 5,25. Neste trabalho foi encontrada razão $\mathrm{Si} / \mathrm{Al}$ próximas a esses valores.

$\mathrm{Na}$ Tabela IV e $\mathrm{V}$ pode-se observar as razões $\mathrm{Si} / \mathrm{Al}$ encontradas nesse trabalho para as amostras sem calcinar e calcinadas. Observa-se que os resultados são próximos, o que pode ser um indicativo de que as impurezas, provavelmente, não contêm $\mathrm{Si}$ ou Al, uma vez que a composição está próxima à da Clinoptilolita. Esses valores de razão $\mathrm{Si} / \mathrm{Al}$ são típicos aos encontrados em outras amostras de Clinoptilolita (SHINZATO et al., 2008; RIBEIRO; RODRIGUES, 2010; BELVISO et al., 2014).

Tabela IV - Razão Si/Al das amostras antes da calcinação

\begin{tabular}{cc}
\hline Amostra & Razão Si/Al \\
\hline RCB LOT 216 & 4,92 \\
RCB LOT 221 & 4,79 \\
RCN1 & 3,98 \\
RCN2 & 3,74 \\
\hline
\end{tabular}

A Figura 5 e 6 representam as curvas de TG e DTG das Clinoptilolitas. Estas curvas indicam dois eventos térmicos, conforme a presença de dois picos nas curvas de DTG. Esses eventos térmicos são referentes à desidratação e desidroxilação.

De acordo com Kurkuna et al. (2006) e Mansouri et al. (2013), existem na estrutura dos minerais de silicato, como a Clinoptilolita, duas formas de água: água com moléculas intactas e água estrutural (grupos $\mathrm{OH}$ ). A água intacta é eliminada abaixo de $100^{\circ} \mathrm{C}$, enquanto que os grupos hidroxila são removidos a temperaturas $>400^{\circ} \mathrm{C}$.

Segundo Breck (1974), a água estrutural (grupos $\mathrm{OH}$ ) é eliminada da Clinoptilolita após temperatura superior a $360^{\circ} \mathrm{C}$. O mesmo foi observado nesse trabalho, como pode ser notado nas Figuras 5 e 6.

Figura 5 - Curvas de TG das amostras antes da calcinação

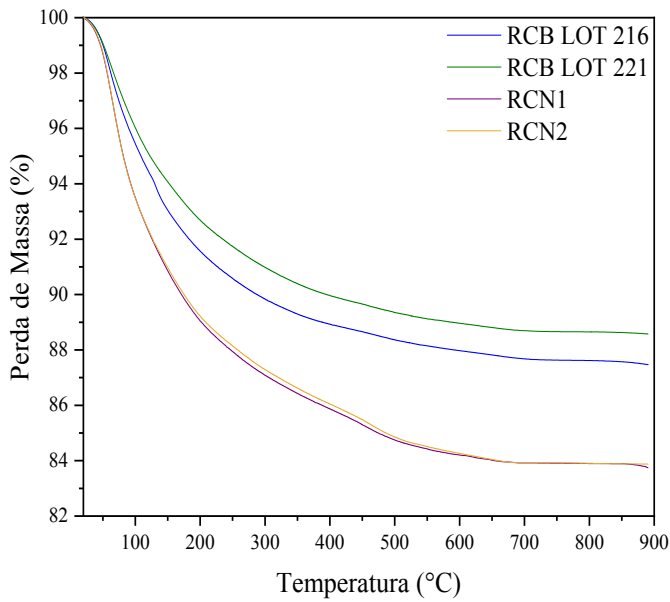

Tabela V - Razão Si/Al das amostras após a calcinação

\begin{tabular}{cc}
\hline Amostra & Razão Si/Al \\
\hline RCB LOT 216 & 5,06 \\
RCB LOT 221 & 5,04 \\
RCN1 & 4,06 \\
RCN2 & 4,06 \\
\hline
\end{tabular}


Tabela VI - Dados termogravimétricos das amostras

\begin{tabular}{ccc}
\hline Amostra & Temperatura máxima $\left({ }^{\circ} \mathbf{C}\right)$ & Perda de massa (\%) \\
\hline RCB LOT 216 & 60,89 & 12,53 \\
RCB LOT 221 & 60,89 & 11,42 \\
RCN1 & 64,92 & 16,25 \\
RCN2 & 64,92 & 16,12 \\
\hline
\end{tabular}

Figura 6 - Curvas de DTG das amostras antes da calcinação

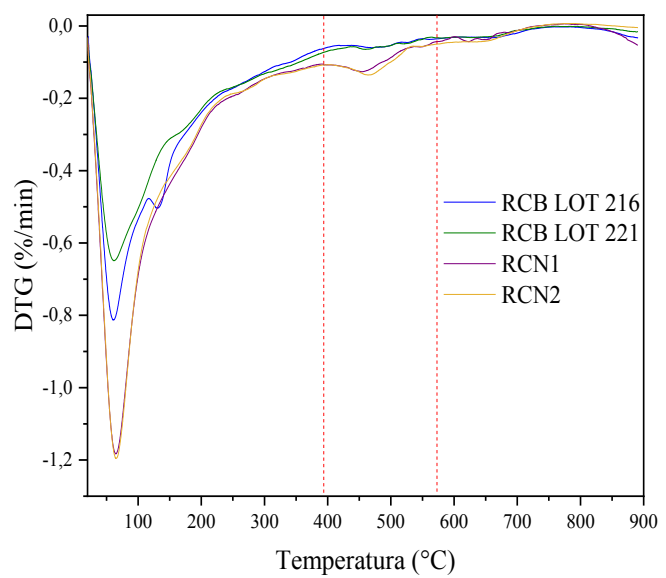

A Tabela VI mostra os dados termogravimétricos das amostras. É possível perceber que as amostras RCB apresentam temperatura máxima igual, assim como as amostras RCN. Porém, as amostras RCB exibem temperatura máxima menor que as amostras $\mathrm{RCN}$, tendo uma diferença de $4,03 \mathrm{C}^{\circ}$. As amostras RCN apresentam maior valores das massas residuais, que exibem percentuais de 16,25 e $16,12 \%$ referentes a RCN1 e RCN2, respectivamente, que podem ser correspon- dentes a à desidratação e desidroxilação e alguma impureza.

Esses resultados então de acordo com os resultados da análise de FRX (Tabela II a V), onde as amostras RCN apresentam menor razão $\mathrm{Si} / \mathrm{Al}$ e maior perda de massa, ou que sugere que a quanto maior a quantidade de $\mathrm{Al}$ mais cátions são necessários para compensar a carga. Uma amostra com mais água pode estar relacionada a ter mais cátions compensando as cargas, pois estes cátions estão solvatados.

\section{Considerações finais}

Os métodos de caracterizações empregados nesse trabalho, foram eficientes para caracterizar e identificar as Clinoptilolitas da Celta Brasil e do Chile. As amostras apresentaram cristalinidade significativa e fases pertinentes à estrutura da zeólita Clinoptilolita.

Os resultados obtidos mostram que as Clinoptilolitas da Celta Brasil, em especial a amostra RCB LOT 221, apresentaram maiores razões $\mathrm{Si} / \mathrm{Al}$, maior estabilidade térmica e menor quantidade de impurezas. 


\section{REFERÊNCIAS}

ARMBRUSTER, T.; GUNTER, M. Crystal Structures of Natural Zeolites. Reviews in Mineralogy and Geochemistry, v. 45, p. 1-67, 2001.

BELVISO, C.; CAVALCANTE, F.; SPARTACO, D.; PALMA, A.; RAGONE, P.;

FIORE, S. Mobility of trace elements in fly ash and in zeolitised coal fly ash. Fuel, v. 144, p. 369379, 2014.

BERNARDI, A.C.C.; MONTE, M.B.M; PAIVA, P.R.P.; WERNECK. C.G.; HAIM, P.G.; POLIDORO, J.C. "Potencial de uso de zeólitas na agropecuária". Documento 85, Embrapa Pecuária Sudeste, 2008, 45p.

BISH, D.; BOAK, J. Clinoptilolite-Heulandite Nomenclature. Reviews In Mineralogy And Geochemistry, v. 45, p. 207-216, 2001.

BLANCO VARELA, M.T.; RAMÍREZ, S.M.; EREÑA, I.; GENER, M., CARMONA, P. "Characterization and pozzolanicity of zeolitic Rocks from two Cuban dposits". Applied Clay Science, v. 33, p. 149, 2006.

BRAGA, A. A. C., MORGON, N. H. Descrições estruturais cristalinas de zeólitas. Química Nova, n.1, v.30, p.178-188, 2007.

BRECK D.W., Zeolite molecular sieves, Wiley, NewYork, 1974.

COLELLA, C.; WISE, W. The Iza Handbook Of Natural Zeolites: a tool of knowledge on the most important family of porous minerals. Microporous And Mesoporous Materials, v. 189, p. 4-10, 2014.

ÇULFAZ, M.; YAGIZ, M. "Ion Exchange properties of natural clinoptilolite: lead-sodium and cádmium-sodium equilibria”. Separation an Purfication Tchnology, 37: 93,2004.

FARJALLAT, J. E. S.; SUGUIO, K. Observações sobre zeolitização em basalto e arenito, Nioaque, Mato Grosso. Bol. Soc. Bras. Geol., v.15, n. 3, p. 51-58, 1996.

GIANETTO, G. P.; MONTES, A. R.; RODRÍGUEZ, G. F. Zeolitas - Características, Propriedades y Aplicaciones Industriales. Editorial Innovación Tecnológica, 2. ed. 2000.

IZA. Commission on Natural Zeolites- Clinoptilolite. Disponível em: http://www.iza-online.org./ natural/Datasheets/Clinoptilolite/clinoptilolite.htm. Acesso em: 3 mar. 2020.

IZA. International Zeolite Association. Disponível em: www.iza-structure.org. Acesso em: 3 mar. 2020 .

KANG, S-J; GASHIRA, K. "Modification off differente grades of Korean natural zeolites for increasing cation exchange capacity". Applied Clay Science, v. 12, p.131, 1997.

KORKUNA O.; LEBODA R.; SKUBISZEWSKA-ZIEMBA J.; VRUBLEVSKA T.; GUNKO V.M.; RYCZKOWSKI J. Structural and physicochemical properties of natural zeolites: clinoptilolite and mordenite. Microporous Mesoporous Mater, v. 87, p. 243, 2006.

KOCAK, Y.; TASCI, E.; KAYA, U. The effect of using natural zeolite on the properties and hydration characteristics of blended cements. Construction And Building Materials, v. 47, p.720$727,2013$. 
MANSOURI, N.; RIKHTEGAR, N.; AHMAD PANAHI, H.; ATABI, F.; SHAHRAKI, B.K.

Porosity, chacaracterization and structural properties of natural zeolite - Clinoptilolite - As a sorbent.

Environment Protection Engineering, v. 39, n.1, p. 139-1532, 2013.

RIBEIRO, K. D. F.; RODRIGUES, S. Uso de zeólitas na redução do teor do cátion níquel de efluentes galvânicos. Cerâmica Industrial, v. 15, p. 5-6, 2010.

SERYOTKIN, Y. High-pressure behavior of heu-type zeolites: x-ray diffraction study of clinoptilolite-Na. Microporous And Mesoporous Materials, v. 235, p. 20-31, 2016.

SHINZATO, M. C.; MONTANHEIRO, T. J.; JANASI, V. A.; FRANCISCO DE ASSIS NEGRI, F. A.; YAMAMOTO, J. K; ANDRADE, S. Caracterização tecnológica das zeólitas naturais associadas às rochas eruptivas da Formação Serra Geral, na região de Piraju-Ourinhos (SP). Revista Brasileira de Geociências, v. 38, n. 3, p. 525-532, 2008.

VIEIRA, L. H.; RODRIGUES, M. V.; MARTINS, L. Cristalização convencional de zeólitas e induzida por sementes. Química Nova, v. 37, n. 9, p. 1515-1524, 2014.

YELIZ, Y. Characterization of two natural zeolites for geotechnical and geoenvironmental applications. Applied Clay Science, v. 50, p. 130-136, 2010. 